\title{
La autoestima, la extraversión y el resentimiento como variables predictoras del bienestar subjetivo*
}

\section{Self-Esteem, Extraversion and Resentment as Predictors of Subjective Well-Being}

\author{
JaVier Murillo MuÑoz \\ Universidad del Valle Sede Palmira, Colombia \\ ORCID: http://orcid.org/0000-0001-8601-526X \\ Manuel Alberto Salazar Castillo \\ Universidad del Valle Sede Palmira, Colombia \\ ORCID: http://orcid.org/0000-0002-3250-3986
}

a Autor de correspondencia. Correo electrónico: Javier.murillo@correounivalle.edu.co

Para citar este artículo: Murillo Muñoz, J., \& Salazar Castillo, M. A. (2019). La autoestima, la extraversión y el resentimiento como variables predictoras del bienestar subjetivo. Universitas Psychologica, 18(1), 1-18. https://doi.org/10.11144/Javeriana.upsy 18-1.aerv

\section{RESUMEN}

Estudio correlacional cuyo objetivo fue establecer la relación de las variables autoestima, extraversión y resentimiento con la satisfacción vital y la felicidad, identificando si las primeras podrían predecir las últimas. La muestra de 1207 residentes en el departamento del Valle del Cauca (Colombia) se obtuvo de manera no probabilística mediante la técnica de bola de nieve. Los resultados mostraron una relación positiva de la autoestima y la extraversión y negativa, del resentimiento, con los dos componentes del bienestar (satisfacción vital y felicidad). Las tres variables emergieron como sus predictoras. Los resultados indicaron una relación más estrecha de la autoestima con la satisfacción vital y de la extraversión y el resentimiento, con la felicidad. Los factores disposicionales (autoestima, extraversión y resentimiento) tuvieron mayor peso que las variables sociodemográficas, en la explicación de varianza.

Palabras clave

bienestar subjetivo; satisfacción vital; felicidad; autoestima; extraversión; resentimiento.

\begin{abstract}
A correlational study with the objective to establish the relationship between the variables: self-esteem, extraversion and resentment with life satisfaction and happiness, identifying if the former could predict the latter. The 1207 resident sample from Valle del Cauca, Colombia, was obtained in a non-probabilistic way, using "the snowball" technique. The results showed a positive relationship between self-esteem and extraversion with the components of well-being. Resentment was negatively correlated with life satisfaction and happiness. The three variables emerged as predictors of well-being. Self-esteem was a stronger correlate of life satisfaction and extraversion and resentment were stronger correlates of happiness. In the explanation of variance, the dispositional factors (self-esteem, extraversion and resentment) had greater weight than the sociodemographic variables.
\end{abstract}


Keywords

subjective well-being; life satisfaction; happiness; self-esteem; extraversion; resentment.

El interés de la psicología social por el estudio del bienestar se ha dirigido principalmente hacia el bienestar subjetivo, concepto que incluye un juicio global de la propia vida llamado satisfacción con la vida (Shin \& Johnson, 1978) y un balance de afectos positivos vs. negativos, llamado felicidad (Triadó, Villar, Solé, \& Osuna, 2005). En la exploración de factores que explican el bienestar subjetivo (Alarcón, 2002; Marrero \& Carballeira, 2011), ocupan un lugar preponderante los llamados rasgos de personalidad, parte distintiva y permanente de una persona, cuya relación con el bienestar subjetivo se ha estudiado principalmente en países de Norte América y Europa, pero poco en países latinoamericanos. Puesto que los primeros son considerados individualistas y los últimos, colectivistas, y se acepta que el bienestar subjetivo se asocia a factores diferentes en sociedades colectivistas comparadas con culturas individualistas (Vielma \& Alonso, 2010), no pueden sencillamente extrapolarse los resultados de los primeros a los segundos. Esta investigación pretende contribuir a llenar este vacío. En el caso particular del resentimiento, variable estudiada en este trabajo junto a la extraversión y la autoestima, la carencia se presenta no solo respecto a Latinoamérica. A pesar de que se trata de un rasgo relativamente permanente y asociado a problemas clínicos, pocos trabajos exploran directamente su relación con el bienestar subjetivo. El resentimiento ha sido abordado desde la perspectiva moral más que desde la óptica científica.

\section{El bienestar subjetivo}

El bienestar subjetivo es definido por Marrero y Carballeira (2011) como la sensación que tiene el individuo de que su vida se desarrolla de acuerdo a sus metas, creencias, valores y deseos, lo que le proporciona una sensación de tranquilidad interior. No obstante, el bienestar subjetivo es un concepto relativo, pues cada persona o cultura otorga un significado distinto a lo que es "estar bien". Según Lu y Gilmour (2004), en Oriente, el bienestar enfatiza las obligaciones del rol y el balance dialéctico, mientras que en las culturas euroamericanas lo que se releva es la responsabilidad personal y el propósito explícito. Larsen y Eid (2008), por su parte, resaltan cómo en culturas individualistas, el bienestar subjetivo se asocia a experimentar emociones y a la autoestima, mientras que en culturas colectivistas, a necesidades económicas satisfechas.

El bienestar subjetivo se conforma de un componente cognitivo llamado satisfacción con la vida -o satisfacción vital, como se le llamará en adelante- y de uno emocional, llamado corrientemente felicidad. La satisfacción vital es definida por Shin y Johnson (1978) como el juicio global que una persona hace de su calidad de vida de acuerdo a sus propios criterios. La felicidad se significa como una experiencia subjetiva resultante del balance entre situaciones de placer y displacer (Triadó et al., 2005). Una persona presentará alto bienestar subjetivo si hay satisfacción con su vida y las emociones positivas predominan sobre las negativas. La felicidad tendría fluctuaciones temporales, mientras la satisfacción vital sería un juicio estable de largo plazo.

Las teorías explicativas del bienestar subjetivo se agrupan básicamente en dos grandes modelos: las teorías de arriba-abajo o top-down y las teorías de abajo-arriba o bottom-up (Bak-Klimek, Karatzias, Elliott, \& Maclean, 2015). Según las primeras, el bienestar se deriva de factores disposicionales y de personalidad (Diener \& Biswas-Diener, 2000), lo cual influye en la reacciones e interpretaciones que las personas tienen frente a circunstancias de vida. Según las segundas, el bienestar se origina en condiciones de vida relacionadas con ingreso, trabajo, estatus marital o educación. Si bien hay evidencia empírica para ambos modelos (Bak-Klimek et al., 2015), esta es mayor para los primeros (Gómez, Villegas de Posada, Barrera, \& Cruz, 2007). Un tercer modelo, la teoría de la felicidad sostenible (Lyubomirsky, Sheldon, \& Schkade, 2005) 
considera tanto los factores disposicionales como las condiciones y circunstancias, además de las actividades intencionales que buscan felicidad. No obstante, para este modelo, los factores disposicionales y las actividades intencionales explicarían la mayor parte de la varianza del bienestar.

\section{Las medidas del bienestar subjetivo}

Entre los instrumentos diseñados para medir el bienestar subjetivo tenemos: The Affect Balance Score o ABS (Bradburn, 1965), escala que pretende capturar el bienestar subjetivo en 10 ítems, obteniendo un balance entre sus afectos positivos y negativos; The Positive and Negative Affect Scale o PANAS (Watson, Clark, \& Tellegen, 1988), que consta de 20 ítems que expresan igualmente dos dimensiones: afectos positivos y afectos negativos, a los que les subyacen representaciones específicas como la ansiedad y la depresión, y cuyo uso en clínica es muy popular; The Oxford Happiness Questionnaire ([OHQ]; Hills \& Argyle, 2002), derivado del Oxford Happiness Inventory $(\mathrm{OHI})$, medida unidimensional de felicidad personal de 29 ítems con siete factores: satisfacción vital, eficacia, sociabilidad/empatía, mentalidad positiva, bienestar, alegría y autoestima, donde el concepto de felicidad va más allá del balance de afectos; The Satisfaction with Life Scale (Diener, Emmons, Larsen, \& Griffin, 1985), popular escala de cinco ítems que mide el componente cognitivo del bienestar subjetivo y, por último, The Subjetive Happiness Scale (Lyubomirsky \& Lepper, 1999), instrumento unidimensional de cuatro ítems diseñado para medir su componente emocional. En esta investigación se aplican las dos últimas escalas.

En Latinoamérica, se pueden resaltar los siguientes instrumentos: La escala Multidimensional para la Medición del Bienestar Subjetivo (EMMSAR) de Anguas y Reyes (Anguas, 2000), que mide en 80 ítems la experiencia emocional positiva y negativa, y el componente afectivo-cognitivo de la vida; la Escala de Bienestar Subjetivo de Vera y Tánori
(2002), que mide la dimensión emocional con seis factores: emociones positivas, emociones negativas, afecto positivo, afecto negativo, expresividad negativa y anticipadores, y la dimensión de satisfacción, con siete factores o dominios: familia, social, amigos, personal, religión, gobierno y economía; finalmente, Alarcón (2006, 2015) diseñó la Escala de Felicidad de Lima, en la que se hallaron cuatro factores: ausencia de sentimiento profundo, satisfacción con la vida, realización personal y alegría de vivir. Dicha escala responde a una definición más amplia de felicidad.

\section{Bienestar subjetivo y autoestima}

La autoestima es definida por Verkuyten y Nekuee (2001) como la evaluación global que una persona hace de sí misma y los sentimientos que acompañan dicha evaluación. Su relación con el bienestar ha encontrado soporte empírico en diferentes estudios previos, entre ellos, Hills y Argyle (2002), Judge, Erez, Bono y Thoresen (2002), Cheng y Furnham (2003) y Marrero y Carballeira (2011), tomando la felicidad todos ellos como el indicador de bienestar, y en los estudios de Rey, Extremera y Pena (2011), Kang, Shaver, Sue, Min y Jing (2003) y Murillo y Molero (2012a, 2012b), quienes utilizaron la satisfacción vital como expresión de bienestar subjetivo. También en Latinoamérica se ha hallado soporte empírico que respalda la relación autoestima-bienestar; San Martín y Barra (2013), por ejemplo, la encontraron entre adolescentes chilenos. La procedencia multicultural de estos trabajos sugiere que se trata de un fenómeno global; sin embargo, hay estudios que arrojan resultados contradictorios, como el de Vera, Córdova y Celis (2009), quienes también en Chile, reportaron una relación negativa entre autoestima y satisfacción vital y una relación no significativa de la primera variable con felicidad, en universitarios.

Son estudios claves que señalan la autoestima como importante predictor del bienestar subjetivo y en especial de la satisfacción vital, los trabajos de Ayyash-Abdo y Alamuddin (2007), 
desarrollado en Líbano; González y Marrero (2017) con adultos mexicanos, en el que se aplicaron los mismos instrumentos de esta investigación; Bak-Klimek et al. (2015), cuyo metaanálisis indicó que la autoestima, junto a otros factores disposicionales y de personalidad, emergía como predictor del bienestar y el trabajo de Diener y Diener (2009), desarrollado en 31 naciones y que mostró una sólida relación de la autoestima con la satisfacción vital.

Se ha comprobado que la relación autoestimabienestar subjetivo es más estrecha en culturas individualistas que en culturas colectivistas, al parecer porque en las primeras, el bienestar se deriva ante todo de logros personales, los cuales se exaltan con mayor énfasis (Oishi, Diener, Lucas, \& Suh, 1999), en oposición a los aspectos relacionales y logros colectivos, más valorados en culturas colectivistas (Murillo \& Molero, 2012b). Una razón esgrimida para la estrecha relación entre la autoestima -que se presume variable causal- y los componentes del bienestar subjetivo, se apoya en la llamada "Teoría de la felicidad sostenible" (Lyubomirsky et al., 2005), según la cual, los factores disposicionales explicarían mejor la varianza del bienestar que los factores circunstanciales, ya que las personas tienden a adaptarse a estos últimos.

\section{Bienestar subjetivo y extraversión}

La extraversión se define como un rasgo estable de personalidad caracterizado por la búsqueda de compañía humana y actividad social, lo cual facilita la comunicación con otras personas y la expresión de emociones positivas. Esta definición, si bien propia, está influenciada por Costa y McCrae (1980), Hills y Argyle (2001) y Eysenck (1967).

La extraversión es factor determinante de bienestar según diversos estudios previos. Dicha asociación tiene mayor soporte con la felicidad que con la satisfacción vital (Alarcón, 2000, 2006; Cheng \& Furnham, 2003; Hills \& Argyle, 2002; Nunes, Hutz, \& Giacomoni, 2009), arrojando incluso resultados contradictorios o no concluyentes en pruebas de regresión, respecto a la última (Alarcón, 2002; Marrero \& Carballeira, 2011; Puente \& Cavazos, 2013). No obstante, se acepta en general que la relación entre extraversión y bienestar es en conjunto sólida. Se pueden considerar estudios claves en este sentido, los trabajos de Alarcón (2000) y de González y Marrero (2017), desarrollados en Latinoamérica y el de Schimmack, Radhakrishnan, Oishi, Dzokoto y Ahadi (2002) en EE. UU., Alemania, Japón, México y Ghana, que dejó ver una relación más estable de la extraversión con el balance hedónico que con la satisfacción vital a través de las culturas. Estos últimos investigadores sugirieron, a partir de sus resultados, que la influencia de factores de personalidad como la extraversión en el componente emocional del bienestar subjetivo es pancultural y la influencia en la satisfacción vital estaría mediada por la cultura. Hallazgos como estos han llevado a varios investigadores a considerar la extraversión como un factor universal de bienestar (Diener, Oishi, \& Lucas, 2003; Larsen \& Eid, 2008). Se argumenta como explicación, que las personas extravertidas tienden a ser sociables y asertivas. Tal hipótesis ha sido soportada, entre otros, por Diener y Seligman (2002), quienes, en una investigación adelantada con estudiantes en Estados Unidos, hallaron, al comparar el $10 \%$ más feliz con el $10 \%$ menos feliz, que los primeros eran más sociables y simpáticos y establecían relaciones más estrechas con otras personas. Los investigadores concluyeron que las buenas relaciones sociales serían necesarias para incrementar el bienestar. Larsen y Eid (2008), por su parte, creen que la extraversión es un buen predictor del bienestar subjetivo porque activa emociones y afectos positivos.

\section{Bienestar subjetivo y resentimiento}

En este trabajo, el resentimiento se define como un proceso interno, arraigado y crónico, que se caracteriza por la acumulación de frustración, dolor, rabia, victimización y atribución de culpabilidad a otra $\mathrm{u}$ otras personas, a quienes se considera responsables de habernos 
causado daño. Por su intensidad y cronicidad, se convierte con frecuencia en un rasgo permanente y patológico de personalidad. El resentimiento implica rumiaciones, hostilidad y el convencimiento de haber sido víctima de injusticias (Worthington, Witvliet, Pietrini, \& Miller, 2007) y un fuerte enojo que no se materializa en acciones "efectivas" de venganza contra quien se percibe culpable (Leal, Meneses, Alarcón, \& Karmelic, 2005; León, Romero, Novara, \& Quesada, 1988; Lersch, 1971; Vázquez, 2013). Esa rabia acumulada suele tener un origen específico, pero tiende a generalizarse en el individuo, afectando emociones, cogniciones y comportamientos. Quizá por ello, León et al. (1988) señalaron que no hay resentimientos parciales sino "personas resentidas".

Sin estudios rigurosos suficientes, se han señalado como factores asociados al resentimiento: la atribución exclusiva de culpabilidad, la sensación de haber sido víctima de experiencias injustas (Allport, 1981) y la imposibilidad de llevar a cabo el acto vindicativo (Leal et al., 2005; Lersch, 1971). Finalmente, se ha asociado el resentimiento a la deprivación relativa (Jans-Beken, Lataster, Leontjevas, \& Jacobs, 2015); las personas al compararse con otros, se perciben en peor situación y sienten que les fue negado determinado bien material o espiritual al que creían tener derecho. A pesar de su carácter lesivo para la salud mental, el concepto de resentimiento es relativamente extraño para los sistemas internacionales de clasificación de trastornos psiquiátricos y psicológicos. En la psicología social la relación resentimiento-bienestar ha sido poco estudiada; la revisión de la literatura solo mostró un estudio empírico que relaciona las dos variables entre personas mayores y que sugiere un impacto negativo de la primera en la segunda (Inga \& Vara, 2006). Otro estudio desarrollado por Quoidbach, Berry, Hansenne y Mikolajczak (2010) con estudiantes en Bélgica, encontró que la rumiación negativa, manifestación típica del resentimiento, se relacionó negativamente con la satisfacción vital, logrando predecirla.
Existen también referencias indirectas a dicho impacto negativo del resentimiento, en Cheng y Furnham (2003) y Kim-Prieto, Diener, Tamir, Scollon y Diener (2005). Más interesantes aún podrían ser los estudios sobre el papel de la gratitud y el perdón en el bienestar (Emmons \& Mishra, 2011; Jans-Beken et al., 2015). En la gratitud, al contrario del resentimiento que activa emociones y pensamientos negativos, hay emociones y pensamientos positivos dirigidos a quienes se atribuyen gestos así mismo positivos hacia nosotros. El perdón permitiría superar emociones negativas propias del resentimiento como la amargura y la hostilidad, generando un impacto positivo en el bienestar (Mkrtchyan, 2014; Worthington et al., 2007).

La relevancia de esta investigación estaría dada: a) por el aporte de evidencia empírica sobre la relación entre autoestima y extraversión con los componentes del bienestar subjetivo en el ámbito latinoamericano, y en especial colombiano; b) el tamaño elevado de la muestra $(\mathrm{N}=1207)$, si bien no probabilística, permite mayor confianza estadística en los resultados; c) de manera especial, y hasta donde la revisión de la literatura indica, sería el primer trabajo en nuestro contexto cultural, que arrojaría soporte empírico sobre la relación negativa del resentimiento con el bienestar subjetivo en diversos grupos etarios.

\section{La presente investigación}

La pregunta de investigación formulada fue: ¿Puede predecirse el bienestar subjetivo a partir de las variables autoestima, extraversión y resentimiento? Por su parte, los objetivos planteados fueron: a) establecer la relación de las variables autoestima, extraversión y resentimiento con la satisfacción vital y la felicidad como componentes del bienestar subjetivo, b) identificar cuales, entre las variables señaladas en el primer objetivo, pueden predecir positiva o negativamente, la satisfacción vital y la felicidad como componentes del bienestar subjetivo y c) establecer comparativamente el peso como predictoras del bienestar subjetivo, 
de las variables sociodemográficas versus las variables psicológicas en estudio.

\section{Hipótesis}

H1: Existe una relación positiva y significativa de la autoestima con los componentes medidos del bienestar subjetivo (satisfacción vital y felicidad). Se espera que la autoestima emerja como variable predictora de dichos componentes.

H2: Existe una relación positiva de la extraversión con los componentes del bienestar subjetivo (satisfacción vital y felicidad). Se espera además que la extraversión emerja como variable predictora de dichos componentes.

H3: Existe una relación negativa del resentimiento con los componentes del bienestar subjetivo (satisfacción vital y felicidad). Se espera además que el resentimiento emerja como variable predictora de dichos componentes.

H4: En conjunto las variables psicológicas del estudio, sean mayores predictoras de los componentes del bienestar subjetivo que las variables sociodemográficas.

\section{Método}

Este estudio forma parte de un trabajo empírico con metodología cuantitativa-correlacional y un muestreo no probabilístico por conveniencia, el cual es frecuentemente utilizado en psicología social, y desarrollado en siete ciudades del Valle del Cauca (Colombia), en el marco de un proyecto financiado por la Vicerrectoría de Investigaciones de la Universidad del Valle y la Universidad del Valle, Sede Palmira.

\section{Participantes}

La muestra recolectada en el año 2015 fue de 1207 participantes, residentes en las ciudades de Cali (30.9 \%), Palmira (25.8 \%), Florida (10.3\%), Candelaria (8.4\%), Buga (8.4 $\%)$, Caicedonia (8 \%) y Cartago (8.2\%). Previamente, se descartaron 75 cuestionarios mal diligenciados (5.8 \% del total). Los criterios de selección de la muestra fueron: a) tener entre 18 y 65 años de edad, etapa en la cual los investigadores presumen hay autonomía legal y social plena de los participantes para decidir por sí mismos su colaboración con el estudio, b) residir en ciudades en los cuales vivieran también los estudiantes universitarios que contribuirían con la recolección de la información, lo cual facilitaba el acceso y c) que deseasen colaborar voluntariamente con el estudio sin contraprestación alguna. El $48.4 \%$ de la muestra estuvo constituido por hombres y el $51.6 \%$, por mujeres. La edad media de los participantes fue de 34 años $(D E=$ 12.1 años) con un rango de 47 (18 a 65 años). Por nivel de estudio, los participantes se distribuyeron así: primaria o secundaria: el $42.6 \%$, intermedios o universitario incompleto: el $41.4 \%$ y profesionales el $16.0 \%$. Respecto a sus ingresos, el $48.6 \%$ reportó menos de $\$ 644000$ mensuales (salario mínimo legal en ese momento), el $21.8 \%$ entre $\$ 645000$ y $\$ 999$ 999, el $19.1 \%$ entre $\$ 1000000$ y \$1 999999 y el $10.5 \%$ más de $\$ 2000000$. En cuanto al estado civil, eran solteros al momento de llenar el cuestionario, el $45.9 \%$; vivían en unión libre, el $26 \%$; casados, el $25.1 \%$; separados, el 2.2 $\%$ y viudos el $0.8 \%$. Finalmente, respecto al número de hijos, el $42.6 \%$ no tenía hijos, el $22.6 \%$ tenía uno, el $21.5 \%$ tenía dos y el 13.3 $\%$ tenía tres o más hijos. Por ocupación, los participantes se dividieron en empleados, el 32 $\%$; independientes, el $21.2 \%$; estudiantes, el 17.1 $\%$; operarios u obreros, el $13.8 \%$; amas de casa, el $6.6 \%$; docentes, el $3.5 \%$; desempleados, el $2.3 \%$ y otros, el $3.5 \%$. La distribución de los participantes por estrato socioeconómico indica que el $17.1 \%$ pertenecía al estrato 1 , el $47.2 \%$ al estrato 2 , el $26.7 \%$ al estrato 3 y el $8.7 \%$ a los estratos 4 y 5 .

\section{Instrumentos}

Las variables de estudio se midieron con un cuestionario tipo Likert de 38 ítems que a manera de batería incluyó todas las escalas psicosociales. El rango de respuestas fue de 1 (total desacuerdo) 
a 5 (total acuerdo). A continuación se describen las escalas.

\section{Satisfacción Vital}

Se usó la escala de satisfacción con la vida de Diener (Diener et al., 1985) en su versión en castellano de Cabañero et al. (2004). Dicha escala consta de cinco ítems. Su fiabilidad original fue de 0.87 y la fiabilidad de su versión en castellano utilizada fue de 0.82 . El alfa de la escala en este cuestionario fue de 0.85 . El análisis factorial arrojó que un solo componente explicó el $62.6 \%$ de la varianza, por lo que se cumplió el criterio de unidimensionalidad. Un ejemplo de sus ítems es: "Las condiciones de mi vida son excelentes".

\section{Felicidad}

Junto con la escala de satisfacción con la vida, son las dos escalas con que se midió el bienestar subjetivo. La felicidad fue medida con tres ítems de la escala de felicidad subjetiva de Lyubomirsky (Lyubomirsky \& Lepper, 1999), que en su versión original consta de cuatro ítems. El ítem 4 fue descartado en esta aplicación por cuanto introdujo confusión entre los participantes, lo que redujo la fiabilidad. La escala fue traducida por los autores de este estudio. Su alfa fue de 0.79. El análisis factorial mostró que un solo componente explicó el $71 \%$ de la varianza, siendo unidimensional. Un ejemplo de sus ítems es: "En comparación con la mayoría de mis amigos y/o compañeros me considero: menos feliz 12345 más feliz".

\section{Autoestima}

Fueron aplicados ocho ítems de la escala de autoestima de Rosenberg (1965) que consta de diez ítems en su versión original. Los dos ítems omitidos lo fueron por introducir confusión entre los participantes en estudios previos adelantados con colombianos (Murillo \& Molero, 2012a, 2012b). Generalmente, esta escala ha arrojado resultados de fiabilidad superiores a 0.8 en diversas aplicaciones. En este estudio, se aplicó la versión en español utilizada por MartínezAntón, Buelga y Cava (2007) cuya alfa fue de 0.72. La fiabilidad de los ocho ítems aplicados fue de 0.79. El análisis factorial dejó ver dos componentes que explican el $58 \%$ de la varianza, pero el primero de ellos explicó el $41 \%$, por lo que la escala es asumida como unidimensional. Un ejemplo de sus ítems es: "En conjunto soy un fracasado".

\section{Extraversión}

Se aplicó una escala de nueve ítems de construcción propia, pero inspirada en Eysenck (1967). Su fiabilidad fue de 0.77. El análisis factorial mostró dos componentes que explican el $50.7 \%$ de la varianza, pero dado que el primero de ellos explicó el 37 \% de esta, se acepta la escala como unidimensional. Ejemplos de sus ítems son: "En las reuniones sociales, soy por lo general un animador" y "Me siento muy cómodo en los grupos".

\section{Resentimiento}

Se usó una escala de trece ítems de construcción propia, cuya fiabilidad arrojó un alfa de 0.84. El análisis factorial dejó ver tres componentes con valores propios mayores a 1 y una explicación de varianza del $55.1 \%$. Se asume, sin embargo, como unidimensional ya que la explicación de varianza del primero fue del $35.4 \%$. Ejemplos de sus ítems son: "Hay ciertas personas a las que de alguna manera me gustaría castigar por lo que me hicieron" y "Deberíamos promover el perdón como un valor muy importante" (invertido).

\section{Procedimiento}

La fiabilidad y la validez de las escalas (alfa de Cronbach y análisis factorial por rotación VARIMAX) se comprobaron mediante prueba piloto. Luego se capacitaron estudiantes universitarios vinculados a diferentes sedes de 
la Universidad del Valle, quienes acompañaron la aplicación del cuestionario, aclarando dudas a los participantes. Un estadístico apoyó constantemente el proceso. Los participantes fueron contactados siguiendo la técnica de "bola de nieve" con el apoyo de los colaboradores. Previamente a la aplicación, se informó a cada participante el propósito y naturaleza de la investigación, la confidencialidad de los datos y el carácter anónimo y voluntario de su participación, lo cual les permitía abandonar o negarse a colaborar con esta. Tras ello, se obtuvo su consentimiento informado. En su mayoría, los cuestionarios se aplicaron de manera personalizada. Responderlos llevó de 10 a 15 minutos.

\section{Análisis estadístico}

Para el análisis estadístico, se realizaron pruebas descriptivas preliminares, con el propósito de hallar la media y la desviación estándar; igualmente, pruebas de fiabilidad del instrumento por alfa de Cronbach y de análisis factorial por rotación VARIMAX. Para la confirmación de las hipótesis, se usó la correlación $\mathrm{r}$ de Pearson y la prueba de regresión jerárquica por el método de pasos sucesivos (dos pasos). En el primer paso, se introdujeron en bloque las variables sociodemográficas, con el propósito de controlarlas al igual que en procura de soporte a H4. En el paso dos, se introdujeron también en bloque las variables psicológicas, buscando el soporte a H1, H2 y H3. Los resultados se analizaron mediante un paquete estadístico SPSS, versión 18.

\section{Resultados}

En la Tabla 1, se observan los estadísticos descriptivos. Los participantes presentaron alta autoestima personal $(M=4.26)$, nivel medio alto de felicidad $(M=3.88)$ y satisfacción vital $(M=$ 3.59), nivel medio de extraversión $(M=3.26)$ y nivel bajo de resentimiento $(M=2.46)$.

\section{Tabla 1}

Escalas aplicadas y sus estadísticos descriptivos (N $=1207$ )

\begin{tabular}{llccc}
\hline \multicolumn{1}{c}{ Escala } & N. $^{\circ}$ items & Media & $\alpha$ & $D E$ \\
\hline Satisfacción vital & 5 & 3.59 & 0.85 & 0.83 \\
Felicidad & 3 & 3.88 & 0.79 & 0.76 \\
Autoestima & 8 & 4.26 & 0.79 & 0.62 \\
Extraversión & 9 & 3.26 & 0.77 & 0.67 \\
Resentimiento & 13 & 2.46 & 0.84 & 0.7 \\
\hline
\end{tabular}

Nota.El rango utilizado para todas las escalas fue de 1 a 5. Las mayores puntuaciones indican mayor conformidad con el constructo medido por la escala correspondiente.

En la Tabla 2, se presentan las correlaciones $r$ de Pearson. Como se esperaba de acuerdo al primer supuesto de $\mathrm{H} 1$, la autoestima se relacionó positivamente con la satisfacción vital y la felicidad. El nivel de significancia fue elevado y semejante en ambos casos $(p<0.001)$. La extraversión presentó igualmente relación positiva y significativa con ambos componentes del bienestar subjetivo $(p<0.001)$, siendo mayor con la felicidad. Esto soporta el primer supuesto de H2. El resentimiento, por otro lado, mostró relación negativa, tanto con la satisfacción vital como con la felicidad $(p<0.001$, en ambos casos), soportando el primer supuesto de $\mathrm{H} 3$. Las tres primeras hipótesis fueron soportadas en su primer supuesto, indicando en todos los casos una relación estrecha de la autoestima, la extraversión y el resentimiento con el bienestar subjetivo, pero que en el caso del resentimiento fue negativa.

\section{Tabla 2}

Correlaciones entre las variables en estudio ( $r$ de Pearson)

\begin{tabular}{|c|c|c|c|c|c|}
\hline Variable & 1 & 2 & 3 & 4 & 5 \\
\hline 1. Satisfacción vital & 1 & $0.46^{* * * *}$ & $0.35^{* * * *}$ & $0.23^{* * *}$ & $-0.25^{* * *}$ \\
\hline 2. Felicidad & & 1 & $0.34^{* * * *}$ & $0.32^{* * * *}$ & $-0.28^{* * *}$ \\
\hline 3. Autoestima & & & 1 & $0.11^{* * * *}$ & $-0.42^{\text {**** }}$ \\
\hline 4. Extraversión & & & & 1 & $-0.07^{*}$ \\
\hline 5. Resentimiento & & & & & 1 \\
\hline
\end{tabular}

$$
\begin{gathered}
\text { Nota. } N=1207 . * p<0.05 \\
* * p<0.01 ; * * * p<0.001
\end{gathered}
$$


La autoestima, la extraversión y el resentimiento como predictores de la satisfacción vital

Establecida la correlación entre la autoestima, la extraversión y el resentimiento con la satisfacción vital, se efectuó, en coherencia con el segundo objetivo, una prueba de regresión jerárquica por el método de pasos sucesivos, dirigida a comprobar si las variables psicológicas señaladas podían ser predictoras de la satisfacción vital y la felicidad. Los resultados pueden observarse en las Tablas 3 y 4 . En uno y otro caso, las variables sociodemográficas fueron introducidas en el paso 1 y con el propósito de controlarlas.. En el paso 2, además de las sociodemográficas, se introdujeron las variables psicológicas (autoestima, extraversión y resentimiento).

\section{Tabla 3}

Predictores de la satisfacción vital

\begin{tabular}{|c|c|c|c|c|}
\hline Predictor & $\begin{array}{c}\mathbf{R}^{2} \\
\text { ajustado }\end{array}$ & $\begin{array}{c}\text { Cambio } \\
\text { en } R^{2}\end{array}$ & $\beta$ & $\mathbf{t}$ \\
\hline Paso $1^{a}$ & 0.108 & & & \\
\hline Ciudad & & & $0.14^{* * *}$ & 4.73 \\
\hline Edad & & & 0.02 & 0.48 \\
\hline Sexo & & & -0.02 & -0.70 \\
\hline Ocupación & & & $-0.10 * *$ & -3.16 \\
\hline $\begin{array}{l}\text { Nivel de } \\
\text { estudios }\end{array}$ & & & $0.08^{*}$ & 2.26 \\
\hline Ingresos & & & $0.13^{* * *}$ & 3.47 \\
\hline Estrato & & & $0.10^{* *}$ & 2.93 \\
\hline Estado civil & & & $0.09^{* *}$ & 2.53 \\
\hline N. ${ }^{\circ}$ de hijos & & & $-0.10^{* *}$ & -2.57 \\
\hline Paso $2^{b}$ & 0.237 & 0.129 & & \\
\hline Ciudad & & & $0.13^{* * *}$ & 4.70 \\
\hline Edad & & & -0.01 & -0.37 \\
\hline Sexo & & & -0.01 & -0.41 \\
\hline Ocupación & & & $-0.08 * *$ & -2.64 \\
\hline $\begin{array}{l}\text { Nivel de } \\
\text { estudios }\end{array}$ & & & 0.03 & 0.85 \\
\hline Ingresos & & & $0.12^{* * *}$ & 3.36 \\
\hline Estrato & & & $0.08^{*}$ & 2.52 \\
\hline Estado civil & & & $0.10^{* *}$ & 2.93 \\
\hline N. ${ }^{\circ}$ de hijos & & & $-0.10^{* *}$ & -2.60 \\
\hline Autoestima & & & $0.26^{* * *}$ & 8.68 \\
\hline Extraversión & & & $0.19 * * *$ & 6.98 \\
\hline Resentimiento & & & $-0.08 * *$ & -2.57 \\
\hline $\mathbf{R}^{2}$ Total & & 0.237 & & \\
\hline
\end{tabular}

Nota.a) Varianza explicada por las variables sociodemográficas $=10.8 \%$, b) varianza explicada por las variables psicológicas $=$ $12.9 \%, c)$ varianza total explicada $=23.7$ \%. $* p<0.05 ; * p<0.01 ; * * p<0.001$.
Tabla 4

Predictores de felicidad

\begin{tabular}{|c|c|c|c|c|}
\hline Predictor & $\begin{array}{c}\mathbf{R}^{2} \\
\text { ajustado }\end{array}$ & $\begin{array}{c}\text { Cambio } \\
\text { en } R^{2}\end{array}$ & $\beta$ & $\mathbf{t}$ \\
\hline Paso 1 ${ }^{a}$ & 0.046 & & & \\
\hline Ciudad & & & 0.05 & 1.53 \\
\hline Edad & & & -0.03 & -0.61 \\
\hline Sexo & & & 0.04 & 1.34 \\
\hline Ocupación & & & $-0.09 * *$ & -2.75 \\
\hline $\begin{array}{l}\text { Nivel de } \\
\text { estudios }\end{array}$ & & & 0.04 & 1.14 \\
\hline Ingresos & & & $0.12 * *$ & 3.16 \\
\hline Estrato & & & $0.08^{*}$ & 2.23 \\
\hline Estado civil & & & 0 & -0.08 \\
\hline N. ${ }^{\circ}$ de hijos & & & -0.02 & -0.41 \\
\hline Paso $2^{a}$ & 0.229 & 0.183 & & \\
\hline Ciudad & & & 0.03 & 0.95 \\
\hline Edad & & & -0.06 & -1.45 \\
\hline Sexo & & & 0.05 & 1.79 \\
\hline Ocupación & & & $-0.07 *$ & -2.34 \\
\hline $\begin{array}{l}\text { Nivel de } \\
\text { estudios }\end{array}$ & & & -0.02 & -0.59 \\
\hline Ingresos & & & 0.1 & 3.04 \\
\hline Estrato & & & 0.05 & 1.69 \\
\hline Estado civil & & & 0 & 0.03 \\
\hline $\mathrm{N} .^{\circ}$ de hijos & & & -0.01 & -0.26 \\
\hline Autoestima & & & $0.21 * * *$ & 7.09 \\
\hline Extraversión & & & $0.28 * * *$ & 10.3 \\
\hline Resentimiento & & & $-0.16^{* * *}$ & -5.2 \\
\hline $\mathbf{R}^{2}$ Total & & 0.229 & & \\
\hline
\end{tabular}

Nota.a) Varianza explicada por las variables sociodemográficas $=4.6 \%$, b) varianza explicada por las variables psicológicas $=$ $18.3 \%, \mathrm{c})$ varianza total explicada $=22.9$ \%. *p $<0.05 ; * p<0.01 ; * * *<0.001$.

Como se observa en las Tablas 3 y 4 , la varianza explicada por las variables sociodemográficas fue para la satisfacción vital del $10.8 \%$ y para la felicidad, del $4.6 \%$. Siete variables sociodemográficas (ingresos, estrato, ciudad, estado civil, ocupación, nivel de estudios y n. - de hijos) emergieron como predictoras de la satisfacción vital en el primer caso, pero solo tres, lo fueron de la felicidad (ocupación, ingresos y estrato).

$\mathrm{Al}$ introducir las variables psicológicas en el segundo paso, estas explicaron el $12.9 \%$ de la varianza de la satisfacción vital (Tabla 3) y el $18.3 \%$ de la varianza de la felicidad (Tabla 4). Las variables psicológicas (autoestima, extraversión y resentimiento) emergieron como predictoras, tanto de la satisfacción vital (Tabla 3) como de la felicidad (Tabla 4), siendo la autoestima y la extraversión predictoras positivas 
y el resentimiento, predictor negativo, en ambos casos, soportándose así el segundo supuesto de $\mathrm{H} 1, \mathrm{H} 2$ y H3. En este segundo paso, seis variables sociodemográficas (ocupación, ingresos, estado civil, $\mathrm{n}^{\circ}$ de hijos, ciudad y estrato) continuaron como predictoras de la satisfacción vital, pero solo una (la ocupación) continuó siéndolo de la felicidad.

En general, el peso predictivo de la autoestima fue mayor respecto a la satisfacción vital que respecto a la felicidad, mientras que el peso predictivo de la extraversión y el resentimiento fue mayor con respecto a la felicidad. Finalmente, el peso predictivo de las variables psicológicas fue comparativamente mayor que el de las sociodemográficas al explicar los componentes del bienestar subjetivo (Tablas 3 y 4), lo cual es más evidente con relación a la felicidad. Dichos resultados apoyan la $\mathrm{H} 4$.

\section{Discusión}

Esta investigación buscó establecer la relación entre las variables autoestima, extraversión y resentimiento con la satisfacción vital y la felicidad, componentes del bienestar subjetivo, identificando cuáles, entre las primeras, podían predecir el bienestar. Se pretendió además, comparar el peso predictivo en el bienestar subjetivo de las variables sociodemográficas versus las variables psicológicas. En el cumplimiento de los objetivos, se aplicaron la correlación de Pearson y la regresión jerárquica por el método de pasos sucesivos.

En congruencia con diversos estudios previos, los resultados de esta investigación mostraron una relación positiva de la autoestima, tanto con la satisfacción vital como con la felicidad, emergiendo la autoestima como variable predictora en ambos casos. La estrecha relación de la autoestima con la satisfacción vital ha sido demostrada ya en diferentes regiones del mundo, sugiriendo que se trata de un fenómeno transcultural, como lo evidencian entre otros, los trabajos de Diener et al. (1985), Judge et al. (2002), Kang et al. (2003), Ayyash-Abdo y Alamuddin (2007), Murillo y
Molero (2012a, $2012 b)$ y Rey et al. (2011). La relación autoestima-felicidad ha sido también demostrada en estudios previos adelantados en diferentes países, entre otros, por Hills y Argyle (2001), Judge et al. (2002), Cheng y Furnham (2003) y Marrero y Carballeira (2011). Que la autoestima muestre un efecto estadístico, tanto con la satisfacción vital como con la felicidad en entornos culturales diversos, sugiere que la relación autoestima-bienestar es un hecho robusto que este estudio con vallecaucanos confirmaría una vez más. En la sociedad colombiana es imperativo cultural exaltar la autoestima como un valor principal como lo ilustra la popular frase "uno tiene que quererse a sí mismo". No resulta extraño que en este trabajo dicha variable sea un predictor significativo del bienestar subjetivo. Según Laca, Verdugo y Guzmán (2005), existe "una actitud cultural" que considera los aspectos relevantes para sentirse satisfecho. Estar satisfecho consigo mismo, es posiblemente una condición importante entre los colombianos para estar satisfecho con la vida; ambas constituyen además juicios globales de naturaleza estable.

El nivel de correlación y predicción entre la autoestima y los componentes cognitivo y emocional del bienestar subjetivo es, a pesar de significativo, menor a 0.4 , lo cual es inferior a lo habitualmente encontrado en países individualistas (véanse Diener \& Diener, 2009; Judge et al., 2002; Oishi et al., 1999). Así, se aportaría evidencia en apoyo a quienes han señalado que la asociación autoestimabienestar subjetivo es significativa en todo tipo de sociedades, pero menos acentuada en las culturas colectivistas (Diener et al., 1985; Larsen \& Eid, 2008). Se debería, posiblemente, como ha sido planteado (Murillo \& Molero, 2016; Oishi et al., 1999), al mayor énfasis que las culturas individualistas hacen del logro personal, que, al fin y al cabo, nutre tanto la autoestima como el bienestar subjetivo.

Se esperaba también una relación positiva y significativa de la extraversión con los componentes del bienestar subjetivo, y que, además, aquella variable emergiese como predictora, tanto de la satisfacción vital como 
de la felicidad. Los resultados respaldaron la hipótesis. La relación significativa entre la extraversión y el bienestar subjetivo ha sido demostrada desde hace décadas en diferentes estudios (Costa \& McCrae, 1980). Que tal resultado se repita como una constante, ha llevado incluso a considerar la primera variable como uno de los pocos predictores universales de la segunda (Diener et al., 2003; Larsen \& Eid, 2008), a pesar de que existen varios estudios con resultados no concluyentes al respecto (Alarcón, 2000; Puente \& Cavazos, 2013).

La extraversión presentó una relación más estrecha con la felicidad que con la satisfacción vital, lo cual es coherente con la literatura predominante (Alarcón, 2000; Hills \& Argyle, 2001; Marrero \& Carballeira, 2011). Se ha intentado explicar de varias maneras el hecho de que la extraversión se asocie con mayor fuerza al componente emocional del bienestar que a su componente cognitivo y sea un mejor predictor de los afectos positivos; se afirma, por ejemplo, que los extravertidos pueden disfrutar de las pequeñas cosas, lo cual genera emociones positivas (Hervas, 2009). Se ha señalado también que la razón estriba en que los extravertidos tienden a buscar actividades que les resulten motivantes. La explicación más plausible, sin embargo, establece una mediación de la socialización (Diener \& Seligman, 2002; Hills \& Argyle, 2001). En efecto, y como lo señalan Hills y Argyle (2001), al ser la extraversión una medida de socialización y siendo además las relaciones sociales un recurso de felicidad, es natural que haya una fuerte conexión entre las dos variables. Adicionalmente, podría considerarse que las personas extravertidas son más espontaneas, y la espontaneidad suele ser expresión de felicidad. Aquella puede ser un canal que permite el fluir y la retroalimentación de la felicidad. No obstante, lo que sigue sin explicarse es si la extraversión es fuente de felicidad, si la felicidad nos hace más extravertidos o ambas cosas.

Se esperaba una relación negativa del resentimiento con los componentes del bienestar subjetivo e igualmente que el resentimiento emergiese como variable predictora. Los resultados son congruentes con la escasa literatura empírica que ha sugerido un impacto negativo del resentimiento y sus manifestaciones en el bienestar (Inga \& Vara, 2006; Quoidbach et al., 2010) y con un mayor número de trabajos que habían advertido desde lo conceptual que las emociones negativas que acompañan el resentimiento menoscaban el bienestar (León et al., 1988; Vásquez, 2013; Worthington et al., 2007). También soporta indirectamente a quienes han planteado un efecto positivo de la gratitud y el perdón, en el bienestar subjetivo (Emmons \& Mishra, 2011; Jans-Beken et al., 2015; Worthington et al., 2007). La gratitud y el perdón son procesos emocionales opuestos al resentimiento. En la primera, se atribuye a que otras personas nos afectan positivamente con su comportamiento, por lo que se generan afectos y emociones positivas hacia ellas, todo lo contrario del resentimiento, donde lo que se atribuye es la culpa por causarnos daño y las emociones que acompañan son negativas. El perdón sustituye emociones negativas por emociones positivas $\mathrm{O}$ neutras (Mkrtchyan, 2014; Worthington, 2006).

En esta investigación, el resentimiento predijo negativamente con mayor fuerza el componente emocional del bienestar subjetivo que su componente cognitivo. El perdón revertiría esas emociones negativas asociadas al resentimiento, generando un efecto positivo en el bienestar. El perdón no es un proceso racional, aunque impacte en este; ocurre en el campo de las emociones, sustituyendo la ira, la amargura, la culpa, la victimización y el dolor, por otras positivas o neutras. En el resentimiento, el deseo de venganza es una necesidad siempre aplazada (Leal et al., 2005; Lersch, 1971); en el perdón, la necesidad de venganza ha perdido su razón de ser y su fuerza; de ahí, su efecto positivo sobre el bienestar, ya que, para una persona resentida, anclada emocional y negativamente en el pasado, dicha carga es abrumadora y desgastante; el perdón la ha liberado de dicha carga. Además, el resentimiento produce un efecto contrario al de la extraversión en la espontaneidad. El resentimiento por lo general inhibe la expresión de emociones positivas, lo cual hace perder espontaneidad. 
Se había postulado que las variables psicológicas del estudio serían mayores predictoras de los componentes del bienestar subjetivo que las variables sociodemográficas. Los resultados avalaron dicha hipótesis a pesar de que solo se consideraron tres (autoestima, extraversión y resentimiento), lo cual ilustra la importancia de ellas en el bienestar. Dichos resultados aportarían evidencia favorable a quienes, desde las teorías de arriba- abajo (Back-Klimek et al., 2015) y aun desde la teoría de la felicidad sostenible (Lyobomirsky et al., 2005), han señalado la fuerte influencia de los factores disposicionales en la varianza explicada del bienestar, al igual que a quienes han atribuido un peso modesto de las variables sociodemográficas en la explicación de la varianza de la felicidad (Alarcón, 2000; Diener, 1994). Si la satisfacción vital constituye un juicio global sobre la vida, poco sujeto a cambios, y la felicidad, un mecanismo de afrontamiento relativamente estable (si bien menos estable que la satisfacción vital), es poco probable que estos dos componentes de bienestar dependan de circunstancias cambiantes.

\section{Conclusiones y principales contribuciones}

Este estudio aporta evidencia acerca de la solidez de la relación positiva entre la autoestima y la extraversión con el bienestar subjetivo; también halló una relación negativa del resentimiento con los componentes del bienestar subjetivo. Esta línea de investigación ha sido poco desarrollada al interior de la psicología social, por lo que el presente trabajo contribuye a ampliar la perspectiva respecto a los factores disposicionales que afectarían negativamente el bienestar. El resentimiento se tornaría en un rasgo lesivo de la personalidad al acumular dolor, frustración, atribución de culpabilidad e ira contenida. La investigación también muestra cómo en conjunto las tres variables psicológicas explicaron mejor el bienestar subjetivo de los participantes que las variables sociodemográficas. Este hallazgo es importante puesto que se trata solo de tres variables y aportaría evidencia útil a quienes han señalado el peso de factores disposicionales en la varianza explicada del bienestar (los modelos teóricos llamados de arriba-abajo (Bak-Klimek et al., 2015). Una conclusión final sería que el bienestar subjetivo se nutre de varios factores y no puede ser explicado de manera aislada por uno solo de ellos, por importante que sea, lo cual soporta el modelo de la felicidad sostenible (Lyubomirsky et al., 2005).

\section{Limitaciones, futuras investigaciones y políticas públicas}

Las limitaciones más importantes de este estudio son: a) que el muestreo no probabilístico utilizado no permite generalizar los resultados, por lo que estos son solo aplicables a los participantes del mismo, b) al tratarse de un estudio correlacional no es posible establecer dirección causal en las relaciones encontradas, c) existe la probabilidad de que, como suele ocurrir con los trabajos empíricos basados en autoinformes, los participantes hayan tenido dificultad para la compresión de los ítems o hayan incurrido en autopresentación positiva, a pesar del intento por reducir el riesgo con el acompañamiento personal de estudiantes universitarios entrenados, además de que la mayoría de las escalas utilizadas se originan en otras culturas y responden a la lógica idiomática y cultural en que surgieron (Schimmack et al., 2002). Tales aspectos constituyen una limitación del instrumento a pesar de los intentos de validación estadística a que se sometió. d) Otra limitación adicional a las enumeradas surge del diseño transeccional de la investigación, que solo da cuenta del objeto de estudio en un momento único, y si bien se trata de variables relativamente estables, estas, no obstante, pueden presentar ciertas variaciones en el tiempo que alteren las conclusiones. Por otra parte, se debe hacer mención de otra limitación importante acerca del instrumento: e) consiste en que este podría no dar cuenta plena de los constructos en estudio. Existen diversas medidas para estudiar el bienestar que suelen tener una relación 
importante entre sí, pero puede no ser suficiente con una de ellas para medir el constructo, al ser complementarias. f) Finalmente, habría que señalar como limitación, que el estudio se ocupó solo de tres variables asociadas al bienestar subjetivo entre las diversas variables disposicionales y de personalidad que pueden impactar el bienestar. La presencia de otras variables de personalidad podría, en una prueba de regresión, alterar el carácter predictivo de las estudiadas en este trabajo.

Con base en los resultados de la investigación, se recomienda a futuros investigadores en el tema que, de ser posible, se establezcan resultados diferenciados para los dos componentes del bienestar subjetivo, o que por lo menos se evite concluir efectos sobre el bienestar subjetivo como un todo, si solo se ha estudiado uno de dichos componentes. En este trabajo, se pudo observar que, si bien la autoestima, la extraversión y el resentimiento predicen tanto la satisfacción vital como la felicidad, no impactan con la misma fuerza el componente cognitivo que el componente emocional. Sería útil también que estudios posteriores hiciesen uso de otros métodos de investigación, ya que, al igual que este, la mayoría de estudios en el tema suelen ser correlacionales. Harían falta ecuaciones estructurales que puedan explorar el papel de otras variables en el bienestar que podrían dar cuenta de una mayor varianza, así como también trabajos experimentales y longitudinales, muy poco contemplados hasta hoy en el estudio del bienestar y que podrían aportar a la causalidad de la relación. Es recomendable igualmente que estudios posteriores incluyan otras medidas del constructo bienestar subjetivo que permitan un mayor contraste (por ejemplo: el balance de emociones positivas y negativas) y que se investigue con otras disciplinas igualmente interesadas en el bienestar como la sociología (Veenhoven, 2008) y la economía, con interés en la relación del bienestar subjetivo con las condiciones materiales de vida. Que el estudio pueda ser replicado en otros países latinoamericanos y aún en otras culturas, previa superación de las limitaciones metodológicas señaladas, permitiría avanzar en dirección de la generalización de resultados.

Se recomienda también a futuros investigadores que incluyan el resentimiento, ya que este rasgo ha sido históricamente ignorado en la investigación psicosocial. Los resultados lo justifican, en especial, en países afectados por un conflicto armado interno prolongado. Esta línea de investigación promete ser de gran utilidad por sí misma, dado su posible impacto negativo en la felicidad, como se ha mostrado en este estudio, pero también como un soporte muy importante para el estudio del perdón y su relación con el bienestar. Los autores de este trabajo opinan que es difícil entender los procesos de perdón y bienestar en víctimas, si no se comprende cómo el resentimiento afecta su satisfacción y su felicidad; al fin y al cabo la esencia del perdón es que libera del resentimiento. Por último, sería recomendable, como sugieren Gómez et al. (2007), que se avance en estudios más concienzudos sobre la relación entre bienestar y culturas individualistas y colectivistas, ya que la globalización podría haber impactado dicha relación hasta el punto de afectar "verdades" aceptadas de tiempo atrás. Por ejemplo, que el bienestar subjetivo se asocia poco a los logros individuales en las culturas colectivistas.

Los hallazgos de esta investigación podrían ser útiles en la aplicación de políticas públicas; los entes gubernamentales podrían focalizar sus esfuerzos en el incremento del bienestar de los ciudadanos, trabajando aspectos asociados de largo plazo como los disposicionales y de personalidad y no solo, como habitualmente se hace, con factores externos y circunstanciales. Sería importante la destinación de mayores recursos para programas de promoción y prevención en salud mental que permitan la detección de rasgos como el resentimiento o la introspección, proclives a tener un impacto negativo en el bienestar. También parecería útil fomentar la socialización como recurso de extraversión, dado su papel comprobado en la felicidad. Esto es importante en una época en la cual las personas tienden a reemplazar las relaciones sociales por el internet. Sería conveniente también que los entes a cargo 
amplíen la perspectiva de la salud mental, sin limitarse a los aspectos clínicos tradicionalmente contemplados en los sistemas de clasificación de trastornos. Ello permitiría, por ejemplo, mirar problemas como el suicidio, desde una perspectiva de largo plazo en su asociación con la insatisfacción vital; un importante porcentaje de quienes hoy se suicidan son personas infelices e insatisfechas a lo largo del tiempo y muchos, además, con serios problemas de autoestima. Harían bien, por otro lado, los entes de salud y Gobierno en mirar desde la misma óptica el muy serio problema del resentimiento social: quienes están afectados por tal problema, no solamente serían menos infelices, sino también dejarían de ser caldo de cultivo de fenómenos lesivos como el fascismo (véase Hitler, 2006) y el paramilitarismo (véase Salas, 2008).

\section{Referencias}

Alarcón, R. (2000). Variables psicológicas asociadas con la felicidad. Persona, 3, 147-157. Recuperado de http://revistas.ulima.edu.pe/index.php/P ersona/article/view/810

Alarcón, R. (2002). Fuentes de felicidad; ¿qué hace feliz a la gente? Revista de Psicología, 20(2), 170-195. Recuperado de http://revistas.pucp.edu.pe/index.php/ps icologia/article/view/3710/3692

Alarcón, R. (2006). Desarrollo de una escala factorial para medir la felicidad. Interamerican Journal of Psychology, 40(1), 99-106. Recuperado de http://www.redalyc .org/articulo.oa? id $=28440110$

Alarcón, R. (2015). La idea de la felicidad. Apuntes de Ciencia EF Sociedad, 5(1), 6-9. h ttps://doi.org/10.18259/acs.2015002

Allport, G. W. (1981). Psicología del amor y el odio. Buenos Aires: Editorial Levitán.

Anguas, A. (2000). El bienestar subjetivo en la cultura mexicana (Tesis doctoral inédita). Universidad Nacional Autónoma de México, México.

Ayyash-Abdo, H., \& Alamuddin, R. (2007). Predictors of subjective well-being among college youth in Lebanon. The Journal of Social Psychology, 147(3), 314-319. https://d oi.org/10.3200/SOCP.147.3.265-284

Bak-Klimek, A., Karatzias, T., Elliott, L., \& Maclean, R. (2015). The determinants of well-being among international economic immigrants: A systematic literature review and meta-analysis. Applied Research Quality Life, 10, 161-188. https://doi.org/10.1007/s 11482-013-9297-8

Bradburn, N. M. (1965). The Affect Balance Scale. Chicago: Aldine Publishing Company.

Cabañero, J. M., Martínez, M. R., Cabrero, J. C., Orts, M. I., Reig, A., \& Tosal, B. (2004). Fiabilidad y validez de la Escala de Satisfacción con la Vida de Diener en una muestra de mujeres embarazadas y puérperas. Psicothema, 16(3), 448-455. Recuperado de http://www.psicothema.co $\mathrm{m} /$ psicothema.asp?id $=3017$

Cheng, H., \& Furnham, A. (2003). Personality, self-esteem, and demographic predictions of happiness and depression. Personality and Individual Differences, 34, 921-942. https://d oi.org/10.1016/S0191-8869(02)00078-8

Costa, P. T., Jr., \& McCrae, R. R. (1980). Influence of extraversion and neuroticism on subjective well-being: Happy and unhappy people. Journal of Personality and Social Psychology, 38, 668-678. https://doi.o $\mathrm{rg} / 10.1037 / 0022-3514.38 .4 .668$

Diener, E. (1994). El Bienestar Subjetivo. Intervención Psicosocial, 3(8), 34-43.

Diener, E., \& Biswas-Diener, R. (2000). New directions in subjective well-being research: The cutting edge. Indian Journal of Clinical Psychology, 27(1), 21-33. Recuperado de ht tps://eddiener.com/articles/1079

Diener, E., \& Diener, M. (2009). Cross-cultural correlates of life satisfaction and selfesteem. En E. Diener (Ed.), Culture and well-being: The collected works of Ed Diener (pp. 71-91). Nueva York: Springer. https:// doi.org/10.1007/978-90-481-2352-0 4

Diener, E., Emmond, R. A., Larsen, R., \& Griffin, S. (1985). Satisfaction with Life Scale. Journal of Personality Assessment, 49(1), 
71-75. https://doi.org/10.1207/s15327752j pa4901_13

Diener, E., Oishi, S., \& Lucas, R. E. (2003). Personality, culture, and subjective wellbeing: Emotional and cognitive evaluations of life. Annual Review of Psychology, 54, 403-425. https://doi.org/10.1146/annurev. psych.54.101601.145056

Diener, E., \& Seligman, M. E. P. (2002). Very happy people. Psychological Science, 13, 81-84. https://doi.org/10.1111/1467-9280. 00415

Emmons, R. A., \& Mishra, A. (2011). Why gratitude enhances well-being: What we know, what we need to know. En K. Sheldon, T. Kashdan \& M. F. Steger (Eds.), Designing the future of positive psychology: Taking stock and oving forward (pp. 248-264). Nueva York: Oxford University Press.

Eysenck, H. J. (1967). The biological basis of personality. Springfield, IL: Charles C. Thomas.

Gómez, V., Villegas de Posada, C., Barrera, F., \& Cruz, J. (2007). Factores predictores de bienestar subjetivo en una muestra colombiana. Revista Latinoamericana de Psicología, 39(2), 311-325. Recuperado de http://www.redalyc.org/articulo.oa?id= 80539208

González, J. A., \& Marrero, R. J. (2017). Determinantes sociodemográficos y personales del bienestar subjetivo y psicológico en población mexicana. Suma Psicológica, 24(1), 59-66. https://doi.org/10. 1016/j.sumpsi.2017.01.002

Hervas, G. (2009). Psicología positiva: una introducción. Revista Interuniversitaria de Formación del Profesorado, 66(3), 23-41. Recuperado de https://www.researchgate.n et/publication/236904237_Psicologia_Posi tiva_Una_introduccion

Hills, P., \& Argyle, M. (2001). Happiness introversion-extroversion and happy introverts. Personality and Individual Differences, 30, 595-608. https://doi.org/10. 1016/S0191-8869(00)00058-1
Hills, P., \& Argyle, M. (2002). The Oxford Happiness Questionnaire: A compact scale for the measurement of psychological wellbeing. Personality and Individual Differences, 33(7), 1073-1082. https://doi.org/10.1016/ S0191-8869(01)00213-6

Hitler, A. (2006). Mi Lucha. Bogotá: Editorial Solar.

Inga, J., \& Vara, A. (2006). Factores asociados a la satisfacción de vida de adultos mayores de 60 años en Lima-Perú. Universitas Psychologica, 5(3), 475-485. Recuperado de http://revistas.javeriana.edu.co/index.p hp/revPsycho/article/view/454

Jans-Beken, L., Lataster, J., Leontjevas, R., \& Jacobs, N. (2015). Measuring gratitude: A comparative validation of the Dutch Gratitude Questionnaire (GQ6) and Short Gratitude, Resentment, and Appreciation Test (SGRAT). Psychologica Belgica, 55(1), 19-31. https://doi.org/10.5334./pb.bd

Judge, T. A., Erez, A., Bono, J. E., \& Thoresen, C. J. (2002). Are measures of self-esteem, neuroticism, locus of control, and generalized self-efficacy indicators of a common core construct? Journal of Personality and Social Psychology, 83(3), 693-710. https://doi.org/10.1037/0022-351 4.83.3.693

Kang, S. H., Shaver, P., Sue, S., Min, K. H., \& Jing, H. (2003). Culture-specific patterns in the prediction of life satisfaction: Roles of emotion, relationship quality, and selfesteem. Personality and Social Psychology Bulletin, 29(12), 1596-1608. https://doi.org /10.1177/0146167203255986

Kim-Prieto, C., Diener, E., Tamir, M., Scollom, C., \& Diener, M. (2005). Integrating the diverse definitions of happiness: A timesequential framework of subjective wellbeing. Journal of Happiness Studies, 6, 261-300. https://doi.org/10.1007/s10902-0 05-7226-8

Laca, F. A., Verdugo, J. C., \& Guzmán, J. (2005). Satisfacción con la vida de algunos colectivos mexicanos: una discusión sobre la psicología del bienestar subjetivo. Revista de Enseñanza e Investigación en Psicología, 
10(2), 325-336. Recuperado de http://www .redalyc.org/pdf/292/29210208.pdf

Larsen, R., \& Eid, M. (Eds.). (2008). Ed Diener and the science of subjective well-being. En The science of subjective well-being (pp. 1-13). Nueva York: The Guilford Press.

Leal, F., Meneses, M., Alarcón, A., \& Karmelic, V. (2005). Agresión y resentimiento en los estudiantes secundarios. Revista de Psicología de la Universidad de Chile, 14(1), 29-39. https://doi.org/10.5354/0719-0581. 2005.17332

León, R., Romero, C., Novara, J., \& Quesada, E. (1988). Una escala para medir el resentimiento. Revista Latinoamericana de Psicología, 20(3), 331-354. Recuperado de http://www.redalyc.org/pdf/805/805203 02.pdf

Lersch, P. (1971). La estructura de la personalidad. Barcelona: Editorial Scientia.

Lu, L., \& Gilmour, R. (2004). Culture and conceptions of happiness: Individual oriented and social oriented SWB. Journal of Happiness Studies, 5, 269-291. https://doi .org/10.1007/s10902-004-8789-5

Lyubomirsky, S., \& Lepper, H. (1999). A measure of subjective happiness: Preliminary reliability and construct validation. Social Intervention Research, 46, 137-155. https://d oi.org/10.1023/A:1006824100041

Lyubomirsky, S., Sheldon, K. M., \& Schkade, D. (2005). Pursuing happiness: The architecture of sustainable change. Review of General Psychology, 9(2), 111-131. https: //doi.org/10.1037/1089-2680.9.2.111

Marrero, R. J., \& Carballeira, M. (2011). Wellbeing and personality: Fact-level analyses. Personality and Individual Differences, 50, 206-211. https://doi.org/10.1016/j.paid.201 0.09 .030

Martínez-Antón, M., Buelga, S., \& Cava, M. J. (2007). La satisfacción con la vida en la adolescencia y su relación con la auto-estima y el ajuste escolar. Anuario de Psicología, 38(29), 293-303. Recuperado de https://www.uv.es/lisis/sofia/sofia_satisfa c.pdf
Mkrtchyan, T. L. (2014). Psychological aspects of resentment and forgiveness. Psychology and Behavioral Sciences, 3(1), 1-15. https://doi.o rg/10.11648/j.pbs.20140301.11

Murillo, J., \& Molero, A. F. (2012a). Factores psicosociales asociados al bienestar de inmigrantes de origen colombiano en España. Psychosocial Intervention, 21(3), 319-329. https://doi.org/10.5093/ in $2012 \mathrm{a} 28$

Murillo, J., \& Molero, A. F. (2012b). La satisfacción vital: su relación con el prejuicio, la identidad nacional, la autoestima y el bienestar material. Acta Colombiana de Psicología, 15(2), 99-108. Recuperado de http://www.redalyc.org/arti culo.oa? id $=79825836005$

Murillo, J. \& Molero, A. F. (2016). Relación entre la satisfacción vital y otras variables psicosociales de migrantes colombianos en Estados Unidos. Revista Colombiana de Psicología, 25(1),15-32. http://doi.org/10.15 446/rcp.v25n1.44308

Nunes, C. H. S., Hutz, C. S., \& Giacomoni, C. H. (2009). Associação entre bem estar subjetivo e personalidade no modelo dos Cinco Grandes fatores. Avaliação Psicológica, 8(1), 99-108. Recuperado de http://www.redalyc.org/articulo.oa?id= 335027279009

Oishi, S., Diener, E., Lucas, R. E., \& Suh, E. M. (1999). Cross-cultural variations in predictors of life satisfaction. Personality and Social Psychology Bulletin, 25, 980-990. http s://doi.org/10.1177/01461672992511006

Puente, R., \& Cavazos, J. (2013). Personality factors affect and autonomy support as predictors of life satisfaction. Universitas Psychologica, 12(1), 41-53. Recuperado de http://www.redalyc.org/articulo.oa?id= 64728729005

Quoidbach, J., Berry, E. V., Hansenne, M., \& Mikolajczak, M. (2010). Positive emotion regulation and well-being: Comparing the impact of eight savoring and dampening strategies. Personality and Individual differences, 49(5), 368-373. https: //doi.org/10.1016/j.paid.2010.03.048 
Rey, L., Extremera, N., \& Pena, M. (2011). Perceived emotional intelligence, selfesteem and life satisfaction in adolescents. Psychosocial Intervention, 20, 227-234. https ://doi.org/10.5093/in2011v20n2a10

Rosenberg, M. (1965). Society and the adolescent child. Princeton, NJ: Princeton University Press.

Salas, A. M. (2008). Resentimiento en el paramilitarismo: análisis en el discurso de Carlos Castaño Gil. Bogotá: Universidad del Rosario.

San Martín, J. L., \& Barra, E. (2013). Autoestima, apoyo social y satisfacción vital en adolescentes. Terapia Psicológica, 31(3), 287-291. https://doi.org/10.4067/S0718-48 082013000300003

Schimmack, U., Radhakrishnan, P., Oishi, S., Dzokoto, V., \& Ahadi, S. (2002). Culture, personality, and subjective wellbeing: Integrating process models of life satisfaction. Journal of Personality and Social Psychology, 82 (4), 582-593. https://doi.org/ 10.1037/0022-3514.82.4.582

Shin, D. C., \& Johnson, D. M. (1978). Avowed happiness as an overall assessment of the quality of life. Social Indicators Research, 5, 475-492. https://doi.org/10.1007/BF0035 2944

Triadó, C., Villar, F., Solé, C., \& Osuna, M. J. (2005). La medida del bienestar en personas mayores: adaptación de la Escala Ryff. Revista de Pedagogía General y Aplicada, 58(3), 347-363. Recuperado de https://ialnet.unirioja.es/servlet/articul $\mathrm{o}$ ? codigo $=1464897$

Vázquez, H. M. (2013). El perdón como práctica para la paz. En Experiencias Educando para la Paz (pp. 5-18). México: Impulsora para el Desarrollo Integral del Ser Humano A. C.

Veenhoven, R. (2008). Sociological theories of subjective well-being. En M. Eid \& R. Larsen (Eds.), The science of subjective wellbeing: A tribute to Ed Diener (pp. 44-61). Nueva York: Guilford.

Vera, J. A., \& Tánori, B. C. (2002). Propiedades psicométricas de un instrumento para medir bienestar subjetivo en la población mexicana. Apuntes de Psicología, 20(1), 63-80. Recuperado de https://www.ciad.mx/archivos/desarroll o/publicaciones/PUBLICACIONES/Produ ccion\%20Academica/Articulos/2002/84.p df

Vera, P., Córdova, N., \& Celis, K. (2009). Optimismo versus autoestima: implicancia para la psicología clínica y la psicoterapia. Revista Argentina de Clínica Psicológica, 18(1), 21-30. Recuperado de http://www.re dalyc.org/pdf/2819/281921800002.pdf

Verkuyten, M., \& Nekuee, S. (2001). Selfesteem, discrimination, and coping among refugees: The moderating role of selfcategorization. Journal of Applied Social Psychology, 31(5), 1058-1075. https://doi.or g/10.1111/j.1559-1816.2001.tb02662.x

Vielma, J., \& Alonso, L. (2010). El estudio del bienestar psicológico subjetivo. Una breve revisión teórica. Educare, 14(49), 265-275. Recuperado de http://www.redalyc.org/pdf/ 356/35617102003.pdf

Watson, D., Clark, L., \& Tellegen, A. (1988). Development and validation of brief measures of positive and negative affect: The PANAS Scale. Journal of Personality and Social Psychology, 54, 1063-1070. https: //doi.org/10.1037//0022-3514.54.6.1063

Worthington, E. (2006). Forgiveness and Reconciliation: Theory and Application. New York: Brunner-Routledge.

Worthington, E. L., Jr., Witvliet, C. V. O., Pietrini, P., \& Miller, A. J. (2007). Forgiveness, health, and wellbeing: A review of evidence for emotional versus decisional forgiveness, dispositional forgivingness, and reduced unforgiveness. Journal of Behavioral Medicine, 30, 291-302. https://doi.org/10.1007=s10865007-9105-8

\section{Notas}

* Artículo de investigación. Estudio adelantado entre marzo de 2015 y marzo de 2016 en el marco del proyecto de investigación "Predictores de bienestar 
Javier Murillo Muñoz, Manuel Alberto Salazar Castillo.

subjetivo en residentes de varios municipios del Valle del Cauca", financiado y desarrollado dentro de la Convocatoria Interna - 2014 de la Vicerrectoría de Investigaciones de la Universidad del Valle (Colombia), para sus sedes regionales (Cód. 9028). 